\title{
Skyrmion-Like Solitons, Topological Quasi-Positroniums, and Soliton-Catalytic Effects in Graphite-Potassium Intercalation Compounds and Metal Surfaces
}

\author{
Ikuzo Kanazawa*, Makoto Saito, Tomoaki Sasaki, Erika Imai \\ Department of Physics, Tokyo Gakugei University, Tokyo, Japan \\ Email: ${ }^{*}$ kanazawa@u-gakugei.ac.jp \\ Received 21 July 2014; revised 16 August 2014; accepted 8 September 2014 \\ Copyright @ 2014 by authors and Scientific Research Publishing Inc. \\ This work is licensed under the Creative Commons Attribution International License (CC BY). \\ http://creativecommons.org/licenses/by/4.0/

(c) (i) Open Access

\begin{abstract}
We have analyzed the narrow components in the positron annihilation angular correlation spectra of graphite-potassium intercalation with the theoretical formula, which is extended from "topological quasi-positronium" model and discusses the relation to the catalytic activity of hydrogens. One mechanism of the soliton-catalytic effect is proposed.
\end{abstract}

\section{Keywords}

Model of Surface Chemical Reactions, Catalysis, Alkali Metal, Positron, Positron Annihilation

\section{Introduction}

It is known that alkali-metal graphite intercalation compounds (AGIC) have catalytic activities for hydrogen layer of the compounds. The behaviors of these compounds have been known to depend on the structure, sort of metals and temperature. Hydrogens are physisorptively accommodated in the molecules in the interstices among the alkali-metal ions in intercalant layers of stage-2 compounds $\mathrm{C}_{24} \mathrm{M}(\mathrm{M}=\mathrm{K}, \mathrm{Rb}$ and $\mathrm{Cs})$ at temperatures below $200 \mathrm{~K}$ [1]-[3]. Chemisorption of hydrogen takes place in both first and second stage compounds at higher temperatures [4] [5]. In order to clarify the mechanism of hydrogen chemisorption in the graphite compounds and properties of hydrogen-absorbed graphite compounds, several studies have been carried out. The change in magnetic susceptibilities as a function of hydrogen content has been investigated for hydrogen-chemisorbed $\mathrm{C}_{8} \mathrm{~K}$ [6]. It has been suggested that dissolved hydrogen in $\mathrm{C}_{8} \mathrm{~K}$ is paramagnetic by means of magnetic resonance stu-

\footnotetext{
*Corresponding author.
}

How to cite this paper: Kanazawa, I., Saito, M., Sasaki, T. and Imai, E. (2014) Skyrmion-Like Solitons, Topological QuasiPositroniums, and Soliton-Catalytic Effects in Graphite-Potassium Intercalation Compounds and Metal Surfaces. Journal of Modern Physics, 5, 1483-1488. http://dx.doi.org/10.4236/jmp.2014.515150 
dies [7]. The studies of means of ESR and electrical conductivity [8] have shown that in $\mathrm{C}_{8} \mathrm{~K}, \mathrm{C}_{24} \mathrm{~K}$, and $\mathrm{C}_{24} \mathrm{Rb}$, the hydride ions are stabilized after the dissociation of the hydrogen molecules into atoms and the subsequent charge transfer, and in $\mathrm{C}_{8} \mathrm{Rb}$ hydrogens are absorbed in the atomic form.

Positron annihilation spectroscopy is a useful method in the study of electronic structures of materials. This spectroscopy has been used in the investigation of electronic structures in graphite and AGICs. The momentum distribution of $\sigma$ and $\pi$-electrons in a graphite crystal has been studied by angular correlation of positron annihilation radiation (ACPAR) [9]-[12], and by Doppler-broadening positron-annihilation radiation (DBPAR) [13]-[15]. ACPAR spectra of $\mathrm{C}_{8} \mathrm{~K}$ and $\mathrm{C}_{24} \mathrm{~K}$ after subtraction of a broad contribution account for annihilation with the graphite $\sigma$ and $\pi$-electrons. Features of the narrow components in ACPAR spectra are in good agreement with the quasi-two dimensional electronic structures, which might correspond to the interlayer state with quasi-two dimensional free electron character parallel to the carbon planes [16]. In addition, hydrogen physisorption and chemisorption effects in AGICs have been studied by DBPAR [17]-[20]. The DBPAR spectra line shape of $\mathrm{C}_{8} \mathrm{~K}$ became sharp through hydrogen chemisorption at $300 \mathrm{~K}$ [17] [18], while the spectral line-shape of $\mathrm{C}_{24} \mathrm{Cs}$ became broad through the physisorption of hydrogen molecules at $77 \mathrm{~K}$ [18] [19]. The intensity of the narrow component of DBPAR spectrum of $\mathrm{C}_{8} \mathrm{Rb}$ was suppressed through hydrogen absorption at $300 \mathrm{~K}$, while it decreased at first and then increased through the absorption at the $373 \mathrm{~K}$ [20]. From the change in DBPAR spectral line-shape of $\mathrm{C}_{8} \mathrm{Rb}$, the hydrogen accommodated in $\mathrm{C}_{8} \mathrm{Rb}$ is consider to be atomic at $300 \mathrm{~K}$ and to be in the form of the hydride ion at $373 \mathrm{~K}$. Recently, the present authors have discussed the mechanism of anomalous magnetic effect due to hydrogen uptake in $\mathrm{C}_{8} \mathrm{RbH}_{\mathrm{x}}$ with the theoretical formula, which is extended from "topological quasi-hydrogen" model. It is suggested that the hydrogen state in C8RbHx might have the Kondo-like property. There exist some problems in the assumption that the narrow component of positron annihilation spectra corresponds to the interlayer state in AGICs. That is, the energy level of the interlayer state in the secondstage AGICs is above Fermi energy $E_{F}$. Furthermore, it looks like that the narrow component is not attributed to the simple positronium $\mathrm{P}_{\mathrm{S}}$ in AGICs, because simple $\mathrm{P}_{\mathrm{S}}$ cannot exist in the metallic state of AGICs.

In this study, we analyze the ACPAR spectra in $\mathrm{C}_{24} \mathrm{~K}$ [21] with the theoretical formula, which is extended from "topological quasi-positronium" model [22] and discuss the origin of the anisotropic narrow components in ACPAR spectra, and relation to the catalytic activity of hydrogens in AGICs.

\section{A Model System and the Soliton-Like Quantum Fluctuation}

The structure of the stage- 2 GIC $\mathrm{C}_{24} \mathrm{~K}$ is shown in Figure 1. In the stage-2 $\mathrm{GIC}_{24} \mathrm{~K}$, the atomic density of potassium metal atoms intercalated between graphite layers is reduced to $2 / 3$ of the density of the close-packed structure in the stage- 1 compound, taking into account the difference in composition between the stage- 1 compound $\mathrm{C}_{8} \mathrm{~K}$ and the stage- 2 compound $\mathrm{C}_{24} \mathrm{~K}$. According to the electronic structure model [16], there coexist quasi-two dimensional graphite $\pi$-bands, bands originating from potassium-metal $s$ electrons, and the electronic

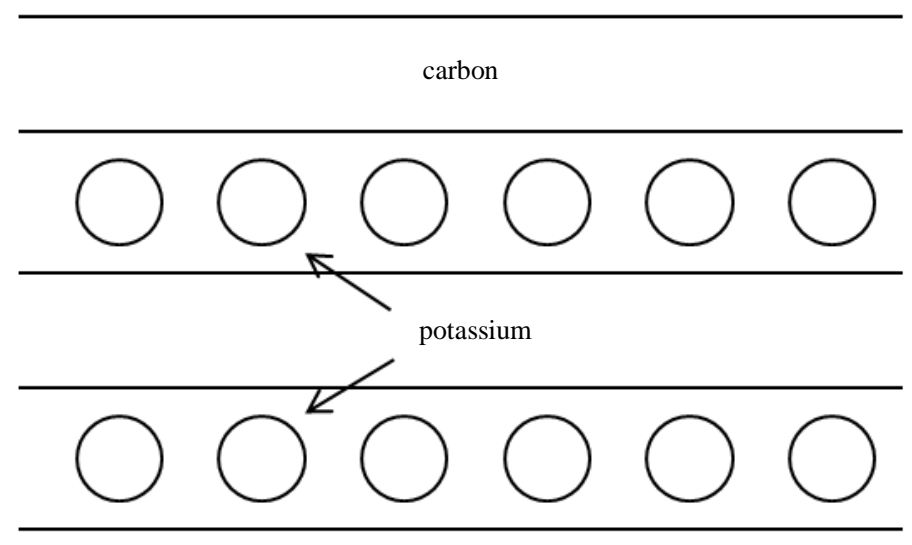

Figure 1. The structure of the stage- 2 GIC $\mathrm{C}_{24} \mathrm{~K}$. The carbon planes and potassium planes have overlapped by turns. 
interlayer state in the stage-2 GIC $\mathrm{C}_{24} \mathrm{~K}$, as shown in Figure 2(a). The Fermi energy $E_{F}$ and the location of the bands are determined by a balance between electronic and lattice energies. The electronic interlayer states were introduced by Posternak et al. [16]. These interlayer states, which exhibit free-electron character parallel to the layers, form a quasi-two dimensional band close to the Fermi energy.

Now, we shall consider the quantum fluctuation for investigation of quasi $(2+1)$ electron state's system. In this study, we propose a kind of quasiparticle "the topological quasi-positronium”, based on the famous quantum fluctuation mechanism by Polyakov [23]. Polyakov [23] explained relatively strong quantum fluctuation in (2+ 1) system as follows. That is, the hedgehog solution [24] [25] in $(3+1)$ system induces instanton-like quantum fluctuation in $(2+1)$ system.

Now, we introduce the Lagrangian density,

$$
\begin{aligned}
L= & \psi^{+}\left(i \partial_{0}-g_{1} T^{a} A_{0}^{a}\right) \psi-\frac{1}{2 m} \psi^{+}\left(i \nabla-g_{1} T^{a} A_{\mu(\neq 0)}^{a}\right)^{2} \psi-\frac{1}{4}\left(F_{\mu \nu}\right)^{2} \\
& +\frac{1}{2}\left(\partial_{\mu} \Phi_{a}-g_{2} \varepsilon_{a b c} A_{\mu}^{a} \Phi_{c}\right)^{2}-\lambda^{2}\left(\Phi_{a} \Phi_{a}-\eta^{2}\right)^{2}
\end{aligned}
$$
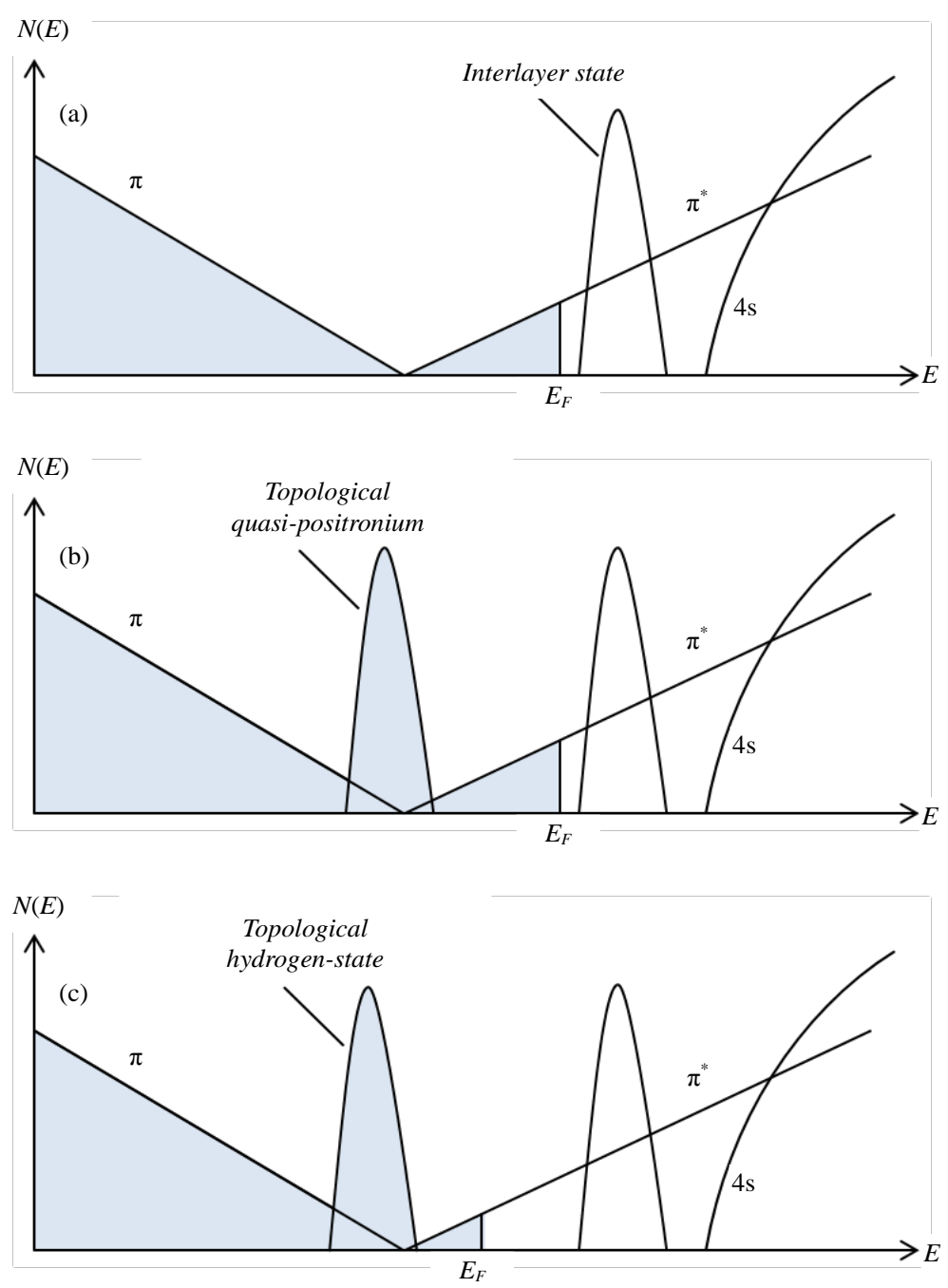

Figure 2. (a), (b), (c) The electronic structure model of (a) alkali-metal GICs and we consider that (b) "topological-quasi-positronium" exists from the result of ACPAR, and (c) absorbed hydrogen alkali-metal GICs. 4s denotes the alkali-metal $s$ band. $\pi$ and $\pi^{*}$ denote graphitic bands. The electronic structure changes by absorbing hydrogen. 
where $\psi$ is the positron field and $\Phi$ is the scalar field. The first and second terms in Equation (1) shows the kinetic energy of positron field and the interaction between the positron field and gauge field. The third term corresponds to the kinetic energy of the gauge field. The fourth term shows the kinetic energy of the scalar field and the interaction between the scalar field and gauge field. The fifth term shows the effective potential.

Here, we set the symmetry breaking $\left\langle 0\left|\Phi_{a}\right| 0\right\rangle=(0,0, \eta) \equiv \phi_{n}$ of the charged scalar field $\Phi_{a}$. We change the field $\Phi_{a}$ with a zero asymptotics at infinity.

$$
\Phi_{a} \rightarrow \phi_{n}+\phi_{a}
$$

Then we enter Equation (2) into Equation (1) and can introduce the effective Lagrangian density as follows.

$$
\begin{aligned}
L_{e f f}= & \psi^{+}\left(i \partial_{0}-g_{1} T^{a} A_{0}^{a}\right) \psi-\frac{1}{2 m} \psi^{+}\left(i \nabla-g_{1} T^{a} A_{\mu(\neq 0)}^{a}\right)^{2} \psi-\frac{1}{4}\left(F_{\mu v}\right)^{2} \\
& +\frac{1}{2}\left(\partial_{\mu} \phi_{a}-g_{2} \varepsilon_{a b c} A_{\mu}^{b} \phi_{c}\right)^{2}+\frac{m_{1}^{2}}{2}\left[\left(A_{\mu}^{1}\right)^{2}+\left(A_{\mu}^{2}\right)^{2}\right]+m_{1}\left[A_{\mu}^{1} \partial_{\mu} \phi_{2}-A_{\mu}^{2} \partial_{\mu} \phi_{1}\right] \\
& +g_{2} m_{1}\left[\phi_{3}\left[\left(A_{\mu}^{1}\right)^{2}+\left(A_{\mu}^{2}\right)^{2}\right]-A_{\mu}^{3}\left[\phi_{1} A_{\mu}^{1}+\phi_{2} A_{\mu}^{2}\right]\right]-\frac{m_{2}^{2}}{2}\left(\phi_{3}\right)^{2}-\frac{m_{2}^{2} g_{2}}{2 m_{1}} \phi_{3}\left(\phi_{a}\right)^{2}-\frac{m_{2}^{2} g_{2}^{2}}{8 m_{1}^{2}}\left(\phi_{a} \phi_{a}\right)^{2}
\end{aligned}
$$

where, $m_{1}=\eta g_{2}$, and $m_{2}=2 \sqrt{2} \lambda \eta$.

Here, it is convenient to choose the following gauge condition.

$$
\phi_{1}=0, \phi_{2}=0, \partial_{\mu} A_{\mu}^{3}=0
$$

When the determinant of the infinitesimal transformation operator in this gauge condition is expressed by det $M$, the generating function of Green function is

$$
Z(J, \eta, \bar{k}, k) \sim \int \exp \left\{i \int\left(L_{e f f}+J \cdot A+\eta \cdot \phi+\bar{k} \cdot \psi+\bar{\psi} \cdot k\right) \mathrm{d}^{3} x\right\} \prod_{x} \operatorname{det} M \mathrm{~d} A \mathrm{~d} \phi \mathrm{d} \psi \mathrm{d} \bar{\psi}
$$

where $J, \eta, \bar{k}$ and $k$ are the sources of the field $A_{\mu}, \phi_{a}, \psi$, and $\bar{\psi}$. Taking into account that $\phi_{n}$ is a kind of the vacuum state $\phi_{3}$ corresponds to the soliton-like-field introduced by t'Hooft [26], based on the disorder parameter [27] [28]. A charged skyrmion-like soliton in quasi $(2+1)$ intercalate state in AGICs might be described as a topological soliton [22] [29] [30]-[32] "Topological quasi-positronium" is regarded as the skyrmion-like soliton, which traps a positron, of the electron-density. "Topological quasi-hydrogen" is regarded as the skyrmion-like soliton, which traps a proton, of the electron-density. Fermion number densities $J_{\text {sky }}^{P}(r)$ and $J_{s k y}^{H}(r)$ of "topological quasi-positronium" and "topological quasi-hydrogen” are represented, respectively, in the case of the SU(2) pseudospin-like skyrmion configuration [30]-[32].

$$
\begin{aligned}
J_{s k y}^{P}(r)=\left|\phi_{3}\right|^{2} & =\frac{1}{\pi} \frac{4 \xi_{p}^{2}}{\left[r^{2}+4 \xi_{p}^{2}\right]^{2}} . \\
J_{s k y}^{H}(r)=\left|\phi_{3}\right|^{2} & =\frac{1}{\pi} \frac{4 \xi_{H}^{2}}{\left[r^{2}+4 \xi_{H}^{2}\right]^{2}} . \\
\xi_{H} & \sim \frac{1}{2} \xi_{P} .
\end{aligned}
$$

Then $J_{s k y}^{P}=\left|\phi_{3}\right|^{2}$ is to be thought to be the induced soliton-like electron density in quasi $(2+1)$ system. Really the quasi $(2+1)$ system is derived from the instanton-like fluctuation of these solitons [33]. Figure 3(a) shows the positron annihilation spectrum for pair-momentum $p$ perpendicular to the crystallographic $c$-axis $(P \perp c)$ in $\mathrm{C}_{24} \mathrm{~K}$ [21]. Assuming $p$-distribution of the narrow component $(p \perp c)$ of the positron annihilation spectrum is isotropic, $p$-distribution of the narrow component $(p \perp c)$ is shown in Figure 3(b). Figure 3(c) shows the autocorrelation function of the electron-wave function perpendicular to the $c$-axis, which is fourier-transformed from the narrow component for pair-momentum p perpendicular to the $c$-axis. The solid line in Figure 3(c) shows the function $J_{s k y}^{P}(r)$. The value of $\xi_{P}$ is estimated to be $\sim 2.1 \AA$. The dotted line in Figure 3(c) shows the function $J_{\text {sky }}^{P}(r)$ with $\xi_{H} \sim 1.0 \AA$, approximately. 

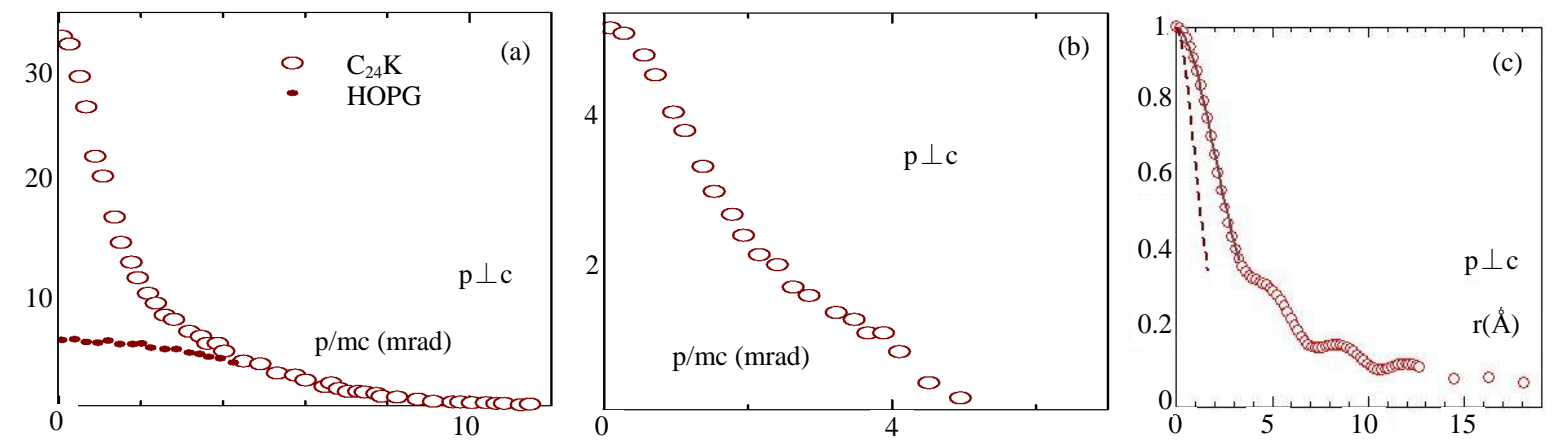

Figure 3. (a) The positron annihilation spectrum for pair-momentum p perpendicular to the $c$-axis in $\mathrm{C}_{24} \mathrm{~K}$ [21]. (b) p-distribution of the narrow component perpendicular to the $c$-axis. (c) The autocorrelation function of the electron-wavefunction perpendicular to the $c$-axis. The solid line in Figure 3(c) shows the function $J_{\text {sky }}^{P}(r)$ with $\xi_{P} \sim 2.1 \AA$. The dotted line in Figure 3(c) shows the function $J_{\text {sky }}^{P}(r)$ with $\xi_{H} \sim 1.0 \AA$.

Here we shall introduce one mechanism of the soliton-catalytic effect as follows. The effective lagrangian for non-abelian gauge fields $A_{\mu}$ in the presence of a soliton $\phi_{3}$ is

$$
L_{\text {eff }}=\frac{1}{16 \pi} \int \mathrm{d}^{3} x \frac{\phi_{3}\left(x_{3}\right)}{\left|\phi_{3}\left(x_{3}\right)\right|} \varepsilon^{\mu \nu \alpha} \operatorname{tr}\left(A_{\mu} \partial_{v} A_{\alpha}+\frac{2}{3} A_{\mu} A_{2} A_{\alpha}\right)
$$

where the $x_{3}$ is parallel to $c$-axis and the plane $\left(x_{1}, x_{2}\right)$ is perpendicular to $c$-axis. The corresponding variation of $L_{\text {eff }}$ under an infinitesimal gauge transformation is

$$
\begin{aligned}
\delta_{\omega} L_{e f f} & =\frac{1}{16 \pi} \int \mathrm{d}^{3} x \frac{\phi_{3}\left(x_{3}\right)}{\left|\phi_{3}\left(x_{3}\right)\right|} \varepsilon^{\mu v \alpha} \partial_{\mu} \operatorname{tr}\left(\omega \partial_{r} A_{\alpha}\right) \\
& \sim-\frac{1}{4 \pi} \int \mathrm{d}^{3} x \varepsilon^{a b} \operatorname{tr}\left(F_{a b} \omega\right)
\end{aligned}
$$

This shows parity anomaly. That is, the soliton-like electron density in the interlayer state induces parity anomaly quantizely. It is suggested that the soliton-like electron density in the interlayer state might induce the parity change, from the bonding electron wave function to the anti-bonding one, in the hydrogen-molecule in the interlayer in AGICs to cancel the parity anomaly.

\section{Conclusion}

We have analyzed the narrow component in the positron annihilation angular correlation spectra in the second stage graphite-potassium intercalation $\mathrm{C}_{24} \mathrm{~K}$ with the theoretical formula extended from "the topological quasipositronium" model and have discussed the relation to the catalytic activity of hydrogens in AGICs.

\section{References}

[1] Watanabe, K., Soma, M., Onishi, T. and Tamaru, K. (1971) Nature, 233, 160-161. http://dx.doi.org/10.1038/physci233160a0

[2] Lagrange, P., Metrot, A. and Herold, C.R. (1972) Académie des Sciences, Paris C, 275, 765.

[3] Watanabe, K., Kondou, T., Soma, M., Onishi, T. and Tamaru, K. (1973) Proceedings of the Royal Society A, 333, 5167.

[4] Kondou, T., Inokuchi, H. and Wakayama, N. (1965) The Journal of Chemical Physics, 43, 3766. http://dx.doi.org/10.1063/1.1696553

[5] Inokuchi, H., Wakayama, N., Kondou, T. and Mori, Y. (1967) The Journal of Chemical Physics, $46,837$. http://dx.doi.org/10.1063/1.1840813

[6] Furdin, G., Lagrange, P., Herold, A. and Zeller, C.R. (1976) Académie des Sciences, Paris C, 282, 563. 
[7] Conard, J., Estrade-Szwarckoph, H., Lauginie, P., Makrini, M., Lagrange, P. and Gnerard, D. (1980) Synthetic Metals, 2, 261-267. http://dx.doi.org/10.1016/0379-6779(80)90055-7

[8] Enoki, T., Sano, M. and Inokuchi, H. (1983) The Journal of Chemical Physics, 78, 2017. http://dx.doi.org/10.1063/1.444949

[9] Colombino, P., Fiscella, B. and Trossi, L. (1963) Nuovo Cimento, 27, 589. http://dx.doi.org/10.1007/BF02784565

[10] Berko, S., Kelly, R.E. and Plaskett, J.S. (1958) Physical Review Letters, 106, 824. http://dx.doi.org/10.1103/PhysRev.106.824

[11] Kanazawa, I., Tanigawa, S., Suzuki, R., Mizuhara, Y., Sano, M. and Inokuchi, H. (1987) Journal of Physics and Chemistry of Solids, 48, 701-705. http://dx.doi.org/10.1016/0022-3697(87)90062-X

[12] Kanazawa, I., Tanigawa, S., Suzuki, R., Sano, M. and Inokuchi, H. (1990) Physical Review B, 42, 11583. http://dx.doi.org/10.1103/PhysRevB.42.11583

[13] Shimotomai, M., Takahashi, T., Doyama, M. and Iwata, T. (1982) Positron Annihilation. Coleman, D.G., Sharma, S.C. and Diana, L.M., Eds., North-Holland, Amsterdam, p. 635.

[14] Cartier, E., Heinrich, F., Pfluger, P. and Güntherodt, H.J. (1981) Physical Review Letters, 46, 272. http://dx.doi.org/10.1103/PhysRevLett.46.272

[15] Cartier, E., Heinrich, F., Gubler, U.M., Pfluger, P., Geiser, V. and Güntherodt, H.J. (1983) Synthetic Metals, 8, 119124.

[16] Posternak, M., Baldereschi, A., Freeman, A.J., Wimmer, E. and Weinert, M. (1983) Physical Review Letters, 50, 761. http://dx.doi.org/10.1103/PhysRevLett.50.761

[17] Murakami, H., Sano, M., Kanazawa, I., Enoki, T., Kurihara, T., Sakurai, Y. and Inokuchi, H. (1985) The Journal of Chemical Physics, 82, 4728. http://dx.doi.org/10.1063/1.448688

[18] Kanazawa, I., Sano, M., Enoki, T., Murakami, H., Sakurai, Y. and Inokuchi, H. (1985) Synthetic Metals, 12, $225-229$. http://dx.doi.org/10.1016/0379-6779(85)90114-6

[19] Sano, M., Kanazawa, I., Murakami, H., Sakurai, Y., Enoki, T. and Inokuchi, H. (1985) Chemical Physics Letters, 122, 143-146. http://dx.doi.org/10.1016/0009-2614(85)85494-4

[20] Murakami, H., Kanazawa, I., Sano, M., Enoki, T. and Inokuchi, H. (1988) Journal of Physics and Chemistry of Solids, 49, 457-460. http://dx.doi.org/10.1016/0022-3697(88)90107-2

[21] Cartier, E., Heinrich, P., Geiser, V. and Güntherodt, H.J. (1985) Positron Annihilation. Jain, P.C., Singra, R.M. and Gopinathan, K.P., Eds., World Science Publisher, Singapore, p. 678.

[22] Kanazawa, I. (1995) Materials Science Forum, 175-178, 695-697. http://dx.doi.org/10.4028/www.scientific.net/MSF.175-178.695

[23] Polyakov, A.M. (1977) Nuclear Physics B, 120, 429-458. http://dx.doi.org/10.1016/0550-3213(77)90086-4

[24] Hooft, G. (1974) Nuclear Physics B, 79, 276-284. http://dx.doi.org/10.1016/0550-3213(74)90486-6

[25] Polyakov, A.M. (1974) JETP Letters, 20, 194.

[26] Hooft, G. (1978) Nuclear Physics B, 138, 1-25. http://dx.doi.org/10.1016/0550-3213(78)90153-0

[27] Kadanoft, L.P. and Ceva, H. (1971) Physical Review B, 3, 3918. http://dx.doi.org/10.1103/PhysRevB.3.3918

[28] Mandelstan, S. (1975) Physical Review D, 11, 3026. http://dx.doi.org/10.1103/PhysRevD.11.3026

[29] Polyakov, A.M. (1987) Gauge Fields and Strings. Harwood Academic Publishers, London.

[30] Wilczek, F. (1980) Fractional Statistics and Anyon and Superconductor. World Science Publisher, Singapore.

[31] Ezawa, Z.F. (1997) Physical Review B, 55, 7771. http://dx.doi.org/10.1103/PhysRevB.55.7771

[32] Manton, N. and Sutcliffe, P. (2007) Topological Solitons. Cambrige University Press, Cambrige.

[33] Kanazawa, I., Yamada, K. and Saito, M. (2012) e-Journal of Surface Science and Nanotechnology, 10, $226-228$. http://dx.doi.org/10.1380/ejssnt.2012.226 
Scientific Research Publishing (SCIRP) is one of the largest Open Access journal publishers. It is currently publishing more than 200 open access, online, peer-reviewed journals covering a wide range of academic disciplines. SCIRP serves the worldwide academic communities and contributes to the progress and application of science with its publication.

Other selected journals from SCIRP are listed as below. Submit your manuscript to us via either submit@scirp.org or Online Submission Portal.
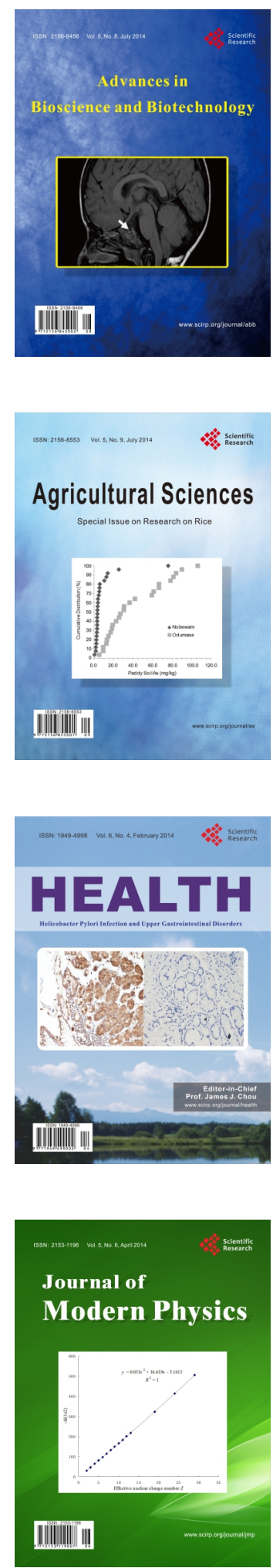
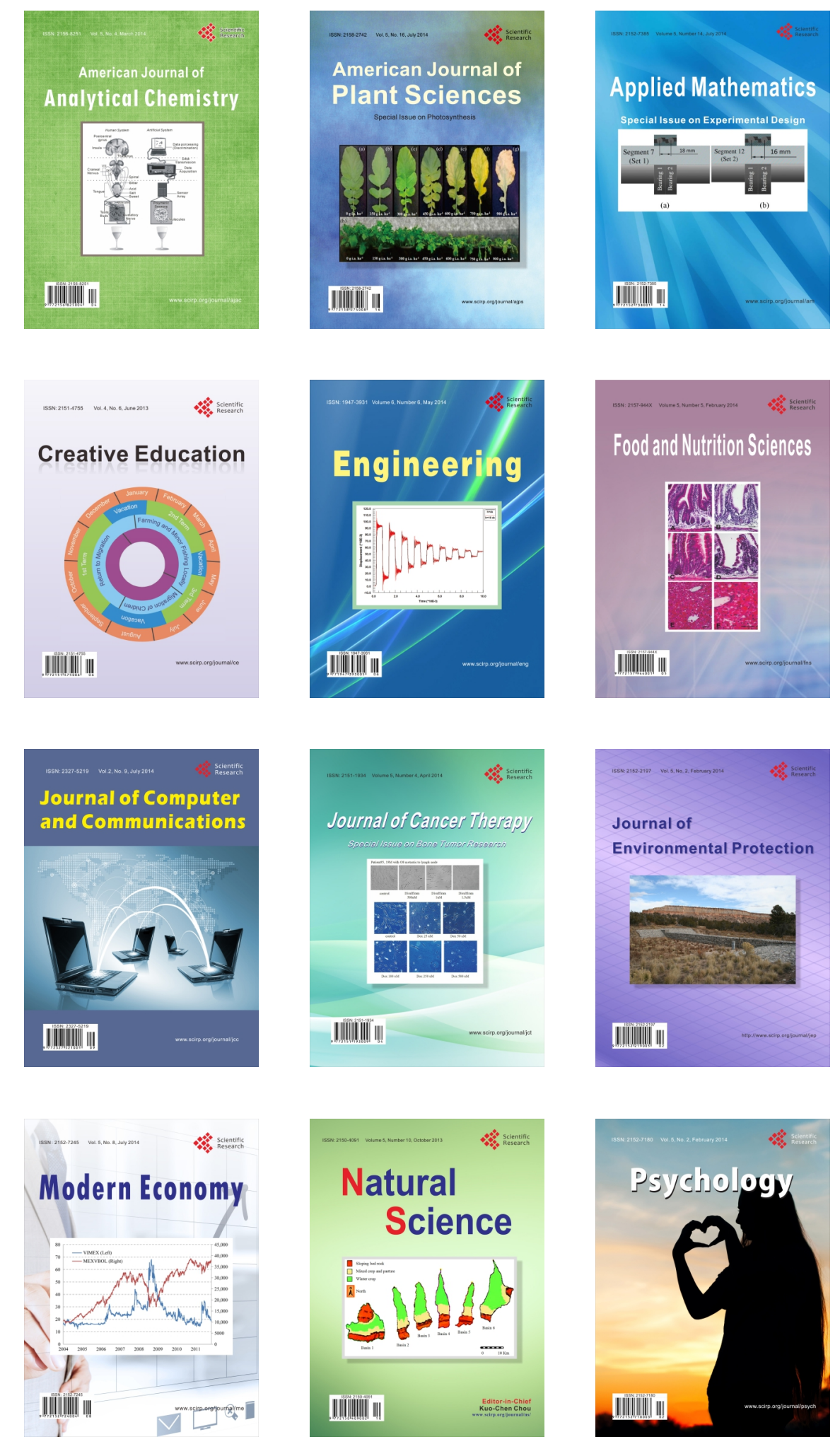